PROCEEDINGS OF THE

AMERICAN MATHEMATICAL SOCIETY

Volume 126, Number 2, February 1998, Pages 595-606

S $0002-9939(98) 04133-1$

\title{
ELEMENTARY ABELIAN 2-GROUP ACTIONS ON FLAG MANIFOLDS AND APPLICATIONS
}

\author{
GOUTAM MUKHERJEE AND PARAMESWARAN SANKARAN
}

(Communicated by Thomas Goodwillie)

\begin{abstract}
Let $\mathcal{N}_{*}$ denote the unoriented cobordism ring. Let $G=(\mathbb{Z} / 2)^{n}$ and let $Z_{*}(G)$ denote the equivariant cobordism ring of smooth manifolds with smooth $G$-actions having finite stationary points. In this paper we show that the unoriented cobordism class of the (real) flag manifold $M=$ $O(m) /\left(O\left(m_{1}\right) \times \cdots \times O\left(m_{s}\right)\right)$ is in the subalgebra generated by $\bigoplus_{i<2^{n}} \mathcal{N}_{i}$, where $m=\sum m_{j}$, and $2^{n-1}<m \leq 2^{n}$. We obtain sufficient conditions for indecomposability of an element in $Z_{*}(G)$. We also obtain a sufficient condition for algebraic independence of any set of elements in $Z_{*}(G)$. Using our criteria, we construct many indecomposable elements in the kernel of the forgetful map $Z_{d}(G) \rightarrow \mathcal{N}_{d}$ in dimensions $2 \leq d<n$, for $n>2$, and show that they generate a polynomial subalgebra of $Z_{*}(G)$.
\end{abstract}

\section{INTRODUCTION}

Let $G=\left(\mathbb{Z}_{2}\right)^{n}, n \geq 2$. Denote by $Z_{*}(G)$ the equivariant cobordism ring of (smooth) closed manifolds with smooth $G$-actions having finite stationary point sets [2], [1]. The cobordism class of a manifold $M$, along with an action $\phi$ of $G$ having finite stationary point set, will be denoted by $[M, \phi]$. Let $R_{q}(G)$ denote the vector space over $\mathbb{Z}_{2}$, with basis the set of isomorphism classes of $\mathbb{R} G$-modules of dimension $q$. If $R_{*}(G)=\sum_{q \geq 0} R_{q}(G)$, then $R_{*}(G)$ is a graded commutative $\mathbb{Z}_{2}$-algebra with unit. The multiplication in $R_{*}(G)$ is given by $[V] \cdot[W]=[V \oplus W]$. One can identify $R_{*}(G)$ with the graded polynomial algebra over $\mathbb{Z}_{2}$ generated by $\widehat{G}=\operatorname{Hom}_{\mathbb{Z}_{2}}\left(G, \mathbb{Z}_{2}\right)$ (cf. [1]).

One has an algebra homomorphism $\eta_{*}: Z_{*}(G) \rightarrow R_{*}(G)$ where $\eta_{*}([M, \phi])=$ $\sum\left[T_{x} M\right]$, the sum being taken over the (finite) set of stationary points of $M$. By a theorem of Stong [11], we know that $\eta_{*}$ is a monomorphism. One has the 'forgetful' homomorphism $\varepsilon_{*}: Z_{*}(G) \rightarrow \mathcal{N}_{*}$, the unoriented cobordism ring, $[M, \phi] \mapsto[M]$. Let $T_{*}^{n}$ denote the subalgebra of $\mathcal{N}_{*}$ generated by $\bigoplus_{i<2^{n}} \mathcal{N}_{i}$. Then tom Dieck [3] has shown that $\operatorname{Im} \varepsilon_{*}=T_{*}^{n}$ (cf. Kosniowski and Stong, section 4 of [5]). In this paper we prove

Theorem 1.1. Let $G\left(m_{1}, \ldots, m_{k}\right)$ denote the flag manifold

$$
O(m) /\left(O\left(m_{1}\right) \times \cdots \times O\left(m_{k}\right)\right),
$$

$m=\sum m_{i}$. Let $2^{n-1}<m \leq 2^{n}$. Then $\left[G\left(m_{1}, \ldots, m_{k}\right)\right] \in T_{*}^{n}$.

Received by the editors July 11, 1996.

1991 Mathematics Subject Classification. Primary 57R75, 57R85.

(C)1998 American Mathematical Society 
The problem of determining which flag manifolds are unoriented boundaries was addressed in [10]. The case of Grassmann manifolds was completely settled in [9], which also considers the case of complex and quaternionic Grassmannians. See also Stong [12]. The above theorem gives perhaps the best known result in general. It is easy to see that if two of the numbers $m_{1}, \ldots, m_{k}$ are equal, then $G\left(m_{1}, \ldots, m_{k}\right)$ admits a fixed point free involution and hence bounds. If $m$ is even, then the above theorem does not yield the best possible result (cf. Theorem 2.1 in [10]). But by remark 2.3(iii) in [10], the general problem of determining which flag manifolds bound has been reduced to the consideration of only the case $m=\sum m_{i}$ is odd, $m_{1}, \ldots, m_{k}$ being distinct.

Theorem 1.1 is proved by exhibiting certain $\left(\mathbb{Z}_{2}\right)^{n}$-actions on $G\left(m_{1}, \ldots, m_{k}\right)$ with finitely many stationary points.

It is well-known that when $n=2, Z_{*}(G)$ is isomorphic to the polynomial algebra with one generator $\left[\mathbb{P}^{2}, \phi\right]$, where the action is given as follows: $t_{1}([x, y, z])=$ $[-x, y, z], t_{2}([x, y, z])=[x,-y, z]$. Here $t_{1}, t_{2}$ denotes a set of generators of $G$. Hence $\varepsilon_{*}: Z_{*}(G) \rightarrow T_{*}^{2}$ is an isomorphism (cf. [1]). However, for $n \geq 3, \varepsilon_{*}$ is not a monomorphism [8] and the structure of $Z_{*}(G)$ is not known.

A $G$-manifold $(M, \phi)$ with finite stationary point set is equivariantly indecomposable if $[M, \phi]$ is an indecomposable element in $Z_{*}(G)$. Clearly, if $[M, \phi] \in Z_{*}(G)$ and $[M] \in \mathcal{N}_{*}$ is indecomposable, then $[M, \phi] \in Z_{*}(G)$ is indecomposable. An important step towards understanding the structure of $Z_{*}(G)$ is to know the indecomposable elements, as they generate $Z_{*}(G)$ as a $\mathbb{Z}_{2}$-algebra. In section 3 we obtain a sufficient criterion for an element in $Z_{*}(G)$ to be indecomposable. Since $T_{*}^{n}$ is a polynomial algebra, and since by tom Dieck's theorem [3] $\operatorname{Im} \varepsilon_{*}=T_{*}^{n}$, it follows that the exact sequence $0 \rightarrow \operatorname{ker} \varepsilon_{*} \rightarrow Z_{*}(G) \rightarrow T_{*}^{n} \rightarrow 0$ splits. Therefore one can clearly 'lift' indecomposable elements from $T_{*}^{n}$ to obtain indecomposable elements in $Z_{*}(G)$. However, we apply our indecomposability criterion to construct indecomposable elements in $Z_{*}(G)$ which belong to $\mathcal{K}_{*}=\operatorname{ker} \varepsilon_{*}$ in each dimension $2 \leq m \leq n$ except possibly in dimension $n$ when $n$ is even. For the precise statement see Theorem 3.6. This is in striking contrast to the situation in the unoriented cobordism ring $\mathcal{N}_{*}$, where there is no generator in dimensions $2^{j}-1$. Note that indecomposable elements in $\mathcal{K}_{*}$ cannot arise by 'lifting' indecomposable elements from $\mathcal{N}_{*}$. We also prove a sufficient criterion for a set of elements in $Z_{*}(G)$ to be algebraically independent and use it to show that suitable indecomposable elements in $\mathcal{K}_{*}$ generate a polynomial subalgebra of $Z_{*}(G)$. In the last section we generalize a result of Conner and Floyd regarding the number of stationary points $x$ such that an irreducible representation occurs at the tangential representation $T_{x} M$ with a given multiplicity.

\section{ACTION OF $\left(\mathbb{Z}_{2}\right)^{n}$ ON FLAG MANIFOLDS}

Let $2 \leq m \leq 2^{n}$, where $n \geq 2$. In this section we shall exhibit certain $G=$ $\left(\mathbb{Z}_{2}\right)^{n}$ actions on the flag manifold $G\left(m_{1}, \ldots, m_{k}\right) \cong O(m) /\left(O\left(m_{1}\right) \times \cdots \times O\left(m_{k}\right)\right)$, $\sum m_{i}=m$. Indeed this is a routine generalization of the actions of $G$ on projective spaces considered by tom Dieck in [3]. We recall the action of $G$ on the Milnor manifold also considered by tom Dieck [3] as it will be needed in section 3 .

Let $\underline{n}=\{1,2, \ldots, n\}$. We regard $\left\{e_{\alpha} \mid \alpha \subset \underline{n}\right\}$ as the 'standard basis' of $\mathbb{R}^{2^{n}}$, with its usual innerproduct. For $1 \leq i \leq n$, let $t_{i}: \mathbb{R}^{2^{n}} \rightarrow \mathbb{R}^{2^{n}}$ be the $\mathbb{R}$-linear map 
defined by

$$
t_{i}\left(e_{\alpha}\right)= \begin{cases}-e_{\alpha} & \text { if } i \in \alpha, \\ e_{\alpha} & \text { if } i \notin \alpha .\end{cases}
$$

Then it is readily checked that $t_{i}^{2}=\mathrm{Id}$, and $t_{i} t_{j}=t_{j} t_{i}$ for $1 \leq i, j \leq n$. Therefore we obtain a linear action of $G$ on $\mathbb{R}^{2^{n}}$.

Lemma 2.1. (i) The decomposition $\mathbb{R}^{2^{n}}=\sum_{\alpha \subset \underline{n}} \mathbb{R} e_{\alpha}$ expresses $\mathbb{R}^{2^{n}}$ as a sum of mutually non-isomorphic irreducible G-submodules of $\mathbb{R}^{2^{n}}$.

(ii) If $V$ is any $G$-submodule of $\mathbb{R}^{2^{n}}$, then $V=\sum_{\alpha \in S} \mathbb{R} e_{\alpha}$ for some $S \subset \mathcal{P}(\underline{n})$ with $\operatorname{dim} V=\# S$.

Proof of (i). It is clear that each $\mathbb{R} e_{\alpha}$ is a $G$-submodule. We only have to prove that if $\alpha \neq \beta$, then $\mathbb{R} e_{\alpha} \nsucceq \mathbb{R} e_{\beta}$. Let $\alpha \neq \beta$, and let $i \in \alpha \Delta \beta$. Say, $i \in \alpha$ and $i \notin \beta$. Then $t_{i} e_{\alpha}=-e_{\alpha}$, and $t_{i} e_{\beta}=e_{\beta}$. This shows that $\mathbb{R} e_{\alpha}$ cannot be isomorphic to $\mathbb{R} e_{\beta}$ as $G$-modules. Part (ii) is an immediate consequence of part (i).

Let $S \subset \mathcal{P}(\underline{n}), \# S=m$. Let $V=\sum_{\alpha \in S} \mathbb{R} e_{\alpha} \subset \mathbb{R}^{2^{n}}$. Since the $G$-action on $\mathbb{R}^{2^{n}}$ preserves innerproduct and since $V$ is a $G$-submodule, we obtain a $G$-action on any flag manifold $G\left(m_{1}, \ldots, m_{k}\right), \sum m_{j}=m$, modelled on $V$. Explicitly, if $\left(A_{1}, \ldots, A_{k}\right) \in G\left(m_{1}, \ldots, m_{k}\right)$, and $t \in G$, then $t\left(A_{1}, \ldots, A_{k}\right)=\left(t A_{1}, \ldots, t A_{k}\right)$. Clearly $\left(A_{1}, \ldots, A_{k}\right)$ is a stationary point if and only if each $A_{j}, 1 \leq j \leq k$, is a $G$-submodule of $V$. We conclude from Lemma 2.1 that there are only finitely many stationary points. We shall denote this action on $G\left(m_{1}, \ldots, m_{k}\right)$ by $\phi_{S}$ or simply by $\phi$ when there is no risk of confusion. Thus $\left[G\left(m_{1}, \ldots, m_{k}\right), \phi_{S}\right] \in Z_{*}(G)$ for every $S \subset \mathcal{P}(\underline{n}), \# S=\sum m_{j}$. In the case of a Grassmann manifold $G_{m, k}$ with $G$-action $\phi_{S}$, the stationary points are $E_{\alpha}=\left\langle e_{\alpha_{1}}, \ldots, e_{\alpha_{k}}\right\rangle$, the span of $e_{\alpha_{1}}, \ldots, e_{\alpha_{k}}$ where $\alpha=\left\{\alpha_{1}, \ldots, \alpha_{k}\right\}$ is any $k$-element subset of $S$.

Next, we recall the action of $G$ on Milnor manifolds considered by tom Dieck [3]. Let $S \subset \mathcal{P}(\underline{n}), \# S=k+1$, and let $T \subset S, \# T=l+1$. Then we can form the product $\left(P^{l} \times P^{k}, \phi_{T} \times \phi_{S}\right)$. Let $H_{l, k}$ be the Milnor manifold

$$
\left\{\left(\left[\sum_{\alpha \in T} x_{\alpha} e_{\alpha}\right],\left[\sum_{\beta \in S} y_{\beta} e_{\beta}\right]\right) \mid \sum_{\alpha \in T} x_{\alpha} y_{\alpha}=0\right\} .
$$

Then $H_{l, k}$ is a $G$-stable submanifold of $\left(P^{l} \times P^{k}, \phi_{T} \times \phi_{S}\right)$. It is obvious that there are only finitely many stationary points for the $G$-action on $H_{l, k}$. We denote this action on $H_{l, k}$ by $\phi_{T, S}$ or simply by $\phi$.

Proof of Theorem 1.1. Since $M=G\left(m_{1}, \ldots, m_{k}\right), m=\sum m_{i} \leq 2^{n}$, was shown to admit a $G=\left(\mathbb{Z}_{2}\right)^{n}$ action with finite stationary point set, it follows from the result of tom Dieck [3] that $[M] \in T_{*}^{n}$. This completes the proof.

Example 2.2. Take $M=G(40,42,45)$. Then the dimension of $M$ is 5370 . The above theorem says that $[M] \in T_{*}^{7}=\mathbb{Z}_{2}\left[x_{2}, x_{4}, \ldots, x_{126}\right]$.

Let $1 \leq k<m \leq 2^{n}$. For any $S \subset \mathcal{P}(\underline{n})$, \#S=m, we obtain an element $\left[G_{m, k}, \phi_{S}\right]$ in $Z_{*}(G), G=\left(\mathbb{Z}_{2}\right)^{n}$. In general distinct choices of $S$ do not necessarily lead to distinct elements $\left[G_{m, k}, \phi_{S}\right]$ in $Z_{*}(G)$. In fact we have the following rather amusing example. 
Example 2.3. Let $m=2^{n}-2,1 \leq k<m, k$ odd. Then for any $S \subset \mathcal{P}(\underline{n})$, $\# S=m$, one has $\left[G_{m, k}, \phi_{S}\right]=0$ in $Z_{*}(G)$.

Proof. First note that $\mathcal{P}(\underline{n})$ has the structure of a Boolean algebra where addition is given by symmetric difference and multiplication, by intersection. For this reason, we write " $\lambda+\mu$ " to mean $\lambda \Delta \mu$ in the proof. Let $S \subset \mathcal{P}(\underline{n}), \# S=2^{n}-2=m$. Let $\alpha, \beta$ be elements of $\mathcal{P}(\underline{n})$ not in $S$. Let $\gamma=\alpha+\beta$. Then $\gamma \neq \varnothing$. It is easy to see that $f: \delta \mapsto \delta+\gamma$ defines a bijection of $S$ onto itself such that $f^{2}=\mathrm{Id}$, and $f$ is fixed point free. It follows that $f$ induces a bijection from the set $\left(\begin{array}{c}S \\ k\end{array}\right)$ of $k$-element subsets of $S$ to itself, denoted by $F$, where $F\left(\left\{\alpha_{1}, \ldots, \alpha_{k}\right\}\right)=\left\{\alpha_{1}+\gamma, \ldots, \alpha_{k}+\gamma\right\}$. Since $k$ is odd, and $f: S \rightarrow S$ is fixed point free, it follows that $F$ is fixed point free.

Recall that the stationary points for the $G$-action $\phi_{S}$ on $G_{m, k}$ are

$$
E_{\alpha}=\left\langle e_{\alpha_{1}}, \ldots, e_{\alpha_{k}}\right\rangle,
$$

where $\alpha=\left\{\alpha_{1}, \ldots, \alpha_{k}\right\} \subset S, \# \alpha=k$. From Lam's [6] description of the tangent bundle of Grassmannians, it is easy to see that the tangential representation $T_{\alpha} G_{m, k}$ of $G$ at $E_{\alpha}$ is isomorphic to

$$
\bigoplus_{\substack{1 \leq i \leq k \\ 1 \leq j \leq m-k}} \mathbb{R} e_{\alpha_{i}} \otimes \mathbb{R} e_{\beta_{j}} \cong \bigoplus_{\substack{1 \leq i \leq k \\ 1 \leq j \leq m-k}} \mathbb{R} e_{\alpha_{i}+\beta_{j}},
$$

where $S \backslash \alpha=\left\{\beta_{1}, \ldots, \beta_{m-k}\right\}$. Since $\alpha_{i}+\beta_{j}=\alpha_{i}+\gamma+\beta_{j}+\gamma=f\left(\alpha_{i}\right)+f\left(\beta_{j}\right)$ for any $\alpha_{i}, \beta_{j} \in S$, it is seen that $T_{\alpha} G_{m, k} \cong T_{F(\alpha)} G_{m, k}$, as $G$-modules. Since $F$ has no fixed point, $\eta_{*}\left[G_{m, k}, \phi_{S}\right]=0$. By Stong's theorem [11], it follows that $\left[G_{m, k}, \phi_{S}\right]=0$.

\section{INDECOMPOSABILITY}

In this section we obtain a sufficient condition for indecomposability of an element in $Z_{*}(G), G=\left(\mathbb{Z}_{2}\right)^{n}$. We apply our criterion to show the existence of indecomposable elements in the kernel $\mathcal{K}_{*}$ of the forgetful homomorphism $\varepsilon_{*}: Z_{*}(G) \rightarrow \mathcal{N}_{*}$. We make use of the equivariant characteristic numbers of a $G$-manifold constructed by tom Dieck [4].

Let $B=\mathbb{Z}_{2}\left[b_{1}, \ldots, b_{m}, \ldots\right]$ be the graded $\mathbb{Z}_{2}$-algebra with $\operatorname{deg} b_{m}=m$, for $m \geq 1$. Let $L_{*}$ denote the $B$-algebra $B\left[\left[y_{1}, \ldots, y_{n}\right]\right]$ of formal power series in $y_{1}, \ldots, y_{n}$ with $\operatorname{deg} y_{i}=1,1 \leq i \leq n$. Let $K_{*}$ denote the fraction field of $L_{*}$. Recall that $R_{*}(G)$ is the polynomial algebra over $\widehat{G}=\operatorname{Hom}_{\mathbb{Z}_{2}}\left(G, \mathbb{Z}_{2}\right)$.

As usual we denote the generators of $G$ by $t_{1}, \ldots, t_{n}$. For $A \subset \underline{n}$, denote by $Y_{A}$ the irreducible representation of $G$ given by the character $\chi_{A} \in \widehat{G}$. Here

$$
\chi_{A}\left(t_{i}\right)= \begin{cases}1 & \text { if } i \in A \\ 0 & \text { if } i \notin A .\end{cases}
$$

Thus $R_{*}(G) \cong \mathbb{Z}_{2}\left[Y_{A} \mid A \subset \underline{n}\right]$. One has the subalgebra $\widetilde{R}_{*}(G)$ of $R_{*}(G)$ generated by $\left\{Y_{A} \mid A \subset \underline{n}, A \neq \varnothing\right\}$. Note that the algebra map $\eta_{*}: Z_{*}(G) \rightarrow R_{*}(G)$ actually has image in $\widetilde{R}_{*}(G)$, and that $\eta_{*}$ is well known to be a monomorphism [11].

Let $\gamma: \widetilde{R}_{*}(G) \rightarrow K_{*}$ denote the $\mathbb{Z}_{2}$-algebra homomorphism defined by $\gamma\left(Y_{A}\right)=$ $\frac{1}{y_{A}} \sum_{r \geq 0} b_{r} y_{A}^{r} \in K_{*}$, where $b_{0}=1, y_{A}=\sum_{i \in A} y_{i}$. Then tom Dieck [4] shows that $\gamma \circ \eta$ is a monomorphism and that $\operatorname{Im}\left(\gamma \circ \eta_{*}\right)$ is contained in $L_{*}$. 
Let $\lambda=\lambda_{1}, \ldots, \lambda_{r}, r \geq 0$, be a non-increasing sequence of natural numbers. We denote by $b_{\lambda}$ the product $b_{\lambda_{1}} b_{\lambda_{2}} \cdots b_{\lambda_{r}} \in B\left(b_{\lambda}=1\right.$ if $\lambda=()$, the empty sequence). For any class $[M, \phi] \in Z_{d}(G)$, one can express $\gamma \eta([M, \phi])$ as $\sum_{|\lambda| \geq 0} \psi_{\lambda}([M, \phi]) \cdot b_{\lambda}$, with $\psi_{\lambda}([M, \phi]) \in \mathbb{Z}_{2}\left(y_{1}, \ldots, y_{n}\right)$ being a homogeneous element of degree $|\lambda|$ $d$, where $|\lambda|=\sum_{j} \lambda_{j}$. The 'integrality theorem' of tom Dieck [4] implies that $\psi_{\lambda}([M, \phi])=0$ if $|\lambda|<d$, and that $\psi_{\lambda}([M, \phi]) \in \mathbb{Z}_{2}\left[y_{1}, \ldots, y_{n}\right]$ if $|\lambda| \geq d$. One can regard $\psi_{\lambda}$ as defining a $\mathbb{Z}_{2}$-linear map $\psi_{\lambda}: Z_{d}(G) \rightarrow \mathbb{Z}_{2}\left[y_{1}, \ldots, y_{n}\right]$. When $|\lambda|=d, \psi_{\lambda}([M, \phi])$ coincides with the Stiefel-Whitney $s$-numbers $s_{\lambda}[M] \in \mathbb{Z}_{2}$ (cf. Kosniowski-Stong [5]). Just as the nonvanishing of $s_{d}(M)$ implies the indecomposability of $[M]$ in $\mathcal{N}_{*}$, one can expect that $\psi_{k}([M, \phi]), k$ any integer greater than $d$, may detect the indecomposability of $[M, \phi] \in Z_{*}(G)$. In fact we have the following

Proposition 3.1. Let $[M, \phi] \in Z_{d}(G)$. Suppose that for some $k>d$, either $\psi_{k}([M, \phi]) \neq 0$ or $\psi_{k-1,1}([M, \phi]) \neq 0$; then $[M, \phi] \in Z_{*}(G)$ is indecomposable.

We need the following lemma to prove the above proposition.

Lemma 3.2. Let $[M, \phi]=\left[M_{1}, \phi_{1}\right] \cdot\left[M_{2}, \phi_{2}\right] \in Z_{d}(G)$, and let $\lambda=\lambda_{1}, \ldots, \lambda_{r}$. Then

$$
\psi_{\lambda}([M, \phi])=\sum_{\lambda=\mu \cdot \nu} \psi_{\mu}\left(\left[M_{1}, \phi_{1}\right]\right) \cdot \psi_{\nu}\left(\left[M_{2}, \phi_{2}\right]\right)
$$

where $\mu \cdot \nu$ denotes juxtaposition of $\mu$ and $\nu$ arranged in the non-increasing order.

Proof of Lemma 3.2. This is a straightforward consequence of the fact that $\gamma$ is an algebra homomorphism. Indeed we have

$$
\begin{aligned}
\sum_{\lambda} \psi_{\lambda}([M, \phi]) b_{\lambda} & =\gamma([M, \phi])=\gamma\left(\left[M_{1}, \phi_{1}\right]\right) \cdot \gamma\left(\left[M_{2}, \phi_{2}\right]\right) \\
& =\left(\sum_{\mu} \psi_{\mu}\left(\left[M_{1}, \phi_{1}\right]\right) b_{\mu}\right)\left(\sum_{\nu} \psi_{\nu}\left(\left[M_{2}, \phi_{2}\right]\right) b_{\nu}\right) \\
& =\sum_{\mu} \sum_{\nu} \psi_{\mu}\left(\left[M_{1}, \phi_{1}\right]\right) \cdot \psi_{\nu}\left(\left[M_{2}, \phi_{2}\right]\right) b_{\mu} b_{\nu} \\
& =\sum_{\lambda}\left\{\sum_{\mu \cdot \nu=\lambda} \psi_{\mu}\left(\left[M_{1}, \phi_{1}\right]\right) \cdot \psi_{\nu}\left(\left[M_{2}, \phi_{2}\right]\right)\right\} b_{\lambda} .
\end{aligned}
$$

Hence, comparing the coefficients of $b_{\lambda}$, we obtain

$$
\psi_{\lambda}([M, \phi])=\sum \psi_{\mu}\left(\left[M_{1}, \phi_{1}\right]\right) \cdot \psi_{\nu}\left(\left[M_{2}, \phi_{2}\right]\right) \in \mathbb{Z}_{2}\left[y_{1}, \ldots, y_{n}\right] .
$$

Proof of Proposition 3.1. Let $\lambda=k-1,1$. If $\lambda=\mu \cdot \nu$, then either $\mu=1$ of $\nu=1$. Note that $Z_{1}(G)=0$, so that for any $[N, \theta] \in Z_{*}(G), \psi_{1}([N, \theta])=0$. By the above lemma it follows that $\psi_{k-1,1}$ vanishes on any decomposable element of $Z_{*}(G)$. Therefore $[M, \phi]$ is indecomposable if $\psi_{k-1,1}([M, \phi]) \neq 0$. The proof for the case $\lambda=k$ is similar.

The following three examples will be used in the proof of Theorem 3.6.

Example 3.3. Let $3 \leq d=2^{k}-1 \leq n$. Let $S=\{\varnothing,\{1\},\{2\}, \ldots,\{d\}\}$. Then $\sigma=\left[P^{d}, \phi_{S}\right]$ is indecomposable in $Z_{*}(G)$. (Note that $\left[P^{d}\right]=0$ in $\mathcal{N}_{*}$.) 
We prove this by showing that $\psi_{d+2}\left(\left[P^{d}, \phi_{S}\right]\right) \neq 0$. Let us write $Y_{i}$ for $Y_{\{i\}}$, and $Y_{i j}$ for $Y_{\{i, j\}}, 1 \leq i, j \leq d$. A straightforward calculation shows that

$$
\eta_{*}(\sigma)=\sum_{i=1}^{d}\left(\prod_{j=1, j \neq i}^{d} Y_{i j}\right) Y_{i}+\prod_{i=1}^{d} Y_{i} .
$$

Let $D=\prod_{k=1}^{d} y_{k} \prod_{1 \leq p<q \leq d}\left(y_{p}+y_{q}\right)$, and for $1 \leq i \leq d$, let

$$
\begin{gathered}
N_{i}=\sum_{p=1}^{d} y_{p}^{d+2}+\sum_{p=1, p \neq i}^{d}\left(y_{i}^{d+1} y_{p}+y_{p}^{d+1} y_{i}\right), \quad C_{i}=\prod_{p=1, p \neq i}^{d} y_{p} \prod_{\substack{1 \leq p<q \leq d \\
p \neq i, q \neq i}}\left(y_{p}+y_{q}\right), \\
N_{0}=\sum_{p=1}^{d} y_{p}^{d+2}, \quad C_{0}=\prod_{1 \leq p<q \leq d}\left(y_{p}+y_{q}\right) .
\end{gathered}
$$

Then $\psi_{d+2}(\sigma)=\frac{1}{D}\left(\sum_{j=0}^{d} N_{j} C_{j}\right)$ and so

$$
D \psi_{d+2}(\sigma)=\sum_{j=0}^{d} N_{j} C_{j} .
$$

One can show that the monomial $y_{1}^{d+1} y_{2}^{d} y_{3}^{d-2} \cdots y_{j}^{d-j+1} \cdots y_{d}$ occurs exactly once on the right hand side of (1). In fact it occurs in $N_{1} C_{1}$ exactly once and in no other $N_{j} C_{j}(j \neq 1)$. Hence $\psi_{d+2}(\sigma) \neq 0$.

Example 3.4. Let $d \leq n, d$ even. As before, let $S=\{\varnothing,\{1\}, \ldots,\{d\}\}$. Let $\sigma=\left[P^{d}, \phi_{S}\right] \in Z_{*}(G)$. Note that $\sigma$ is indecomposable, since $\varepsilon_{*}(\sigma)=\left[P^{d}\right] \in \mathcal{N}_{*}$ is indecomposable. We claim that $\psi_{N}\left(\left[P^{d}, \phi_{S}\right]\right) \neq 0$ for $N=2^{m}, m$ sufficiently large.

To see this, note that

$$
\eta_{*}(\sigma)=\sum_{i=1}^{d}\left(\prod_{j=1, j \neq i}^{d} Y_{i j}\right) Y_{i}+\prod_{j=1}^{d} Y_{j} .
$$

Let $D=\prod_{p=1}^{d} y_{p} \prod_{1 \leq p<q \leq d}\left(y_{p}+y_{q}\right)$ and for $1 \leq i \leq d$, let

$$
N_{i}=y_{i}^{N}+\sum_{p=1, p \neq i}^{d}\left(y_{i}+y_{p}\right)^{N}=\sum_{p=1, p \neq i}^{d} y_{p}^{N},
$$

as $d$ is even and $N$ is a power of 2 , and

$$
C_{i}=\prod_{p=1, p \neq i}^{d} y_{p} \prod_{\substack{1 \leq p<q \leq d \\ p \neq i, q \neq i}}\left(y_{p}+y_{q}\right) .
$$

Let $N_{0}=\sum_{p=1}^{d} y_{p}^{N}$, and $C_{0}=\prod_{1 \leq p<q \leq d}\left(y_{p}+y_{q}\right)$. Then $D \cdot \psi_{N}(\sigma)=\sum_{j=0}^{d} N_{j} C_{j}$. One can show that for $N$ sufficiently large - say $N>\left(\begin{array}{l}d \\ 2\end{array}\right)$ - the monomial $y_{1}^{d-1} y_{2}^{d-2}$ $\cdots y_{d-1} y_{d}^{N}$ occurs in $N_{0} C_{0}$ exactly once and does not occur in any other $N_{j} C_{j}$. Hence the aforementioned monomial survives in $\psi_{N}(\sigma)$. 
Example 3.5. Let $S=\{\varnothing,\{1\}, \ldots,\{k\}\}, T=\{\varnothing,\{1\}, \ldots,\{l\}\}, l<k \leq n$, $k, l$ both even. Recall that one has an action $\phi_{T, S}$ of $G$ on the Milnor manifold $H_{l, k}$. Let $\sigma=\left[H_{l, k}, \phi_{T, S}\right] \in Z_{*}(G)$. Further assume that $\left(\begin{array}{c}k+l \\ k\end{array}\right) \equiv 1(\bmod 2)$, so that $\left[H_{l, k}\right] \in \mathcal{N}_{*}$ is indecomposable. We claim that for $N=2^{\nu}$ sufficiently large, $\psi_{N+1}(\sigma) \neq 0$.

To establish our claim, first note that the normal bundle to the imbedding $H_{l, k} \stackrel{i}{\hookrightarrow}$ $P^{l} \times P^{k}$ is $i^{*}\left(\xi_{l} \otimes \xi_{k}\right)$, where $\xi_{l}$ is the pull-back of the Hopf line bundle over $P^{l}$ via the projection $P^{l} \times P^{k} \rightarrow P^{l}$. Using this it is easy to see that

$$
\eta_{*}(\sigma)=\sum_{\substack{0 \leq i \leq l \\ 0 \leq j \leq k \\ i \neq j}}\left(\prod_{p=0, p \neq i}^{l} Y_{i p} \prod_{q=0, q \neq i, j}^{k} Y_{j q}\right),
$$

with the convention that $Y_{0 p}=Y_{\{p\}}=Y_{p 0}$. Let $D=\prod_{p=1}^{k} y_{p} \prod_{1 \leq p<q \leq k}\left(y_{p}+y_{q}\right)$. For $1 \leq i \leq l, 1 \leq j \leq k$, and $i \neq j$, write

$$
\begin{gathered}
N_{i j}=\sum_{p=l+1}^{k} y_{p}^{N+1}+\sum_{p=1, p \neq i}^{l}\left(y_{i}^{N} y_{p}+y_{i} y_{p}^{N}\right)+\sum_{q=1, q \neq i, j}^{k}\left(y_{j}^{N} y_{q}+y_{j} y_{q}^{N}\right), \\
C_{i j}=\prod_{p=1, p \neq i, j}^{k} y_{p} \cdot \prod_{\substack{1 \leq p<q \leq k \\
q \neq i, j, p \neq i, j}}\left(y_{p}+y_{q}\right) \cdot \prod_{p=l+1}^{k}\left(y_{i}+y_{p}\right) .
\end{gathered}
$$

For $1 \leq j \leq k$, let

$$
\begin{gathered}
N_{0 j}=\sum_{p=l+1}^{k} y_{p}^{N+1}+\sum_{p=1, p \neq j}^{k}\left(y_{j}^{N} y_{p}+y_{j} y_{p}^{N}\right), \\
C_{0 j}=\prod_{\substack{1 \leq p<q \leq k \\
p \neq j, q \neq j}}\left(y_{p}+y_{q}\right) \cdot \prod_{p=l+1}^{k} y_{p} .
\end{gathered}
$$

For $1 \leq i \leq l$, let

$$
\begin{gathered}
N_{i, 0}=\sum_{p=l+1}^{k} y_{p}^{N+1}+\sum_{p=1, p \neq i}^{l}\left(y_{i}^{N} y_{p}+y_{i} y_{p}^{N}\right), \\
C_{i 0}=\prod_{\substack{1 \leq p<q \leq k \\
p \neq i, q \neq i}}\left(y_{p}+y_{q}\right) \cdot \prod_{q=l+1}^{k}\left(y_{i}+y_{q}\right) .
\end{gathered}
$$

Then a routine verification shows that

$$
D \psi_{N+1}(\sigma)=\sum_{\substack{0 \leq i \leq l \\ 0 \leq j \leq k \\ i \neq j}} N_{i j} C_{i j}
$$

Consider the monomial $y_{1} y_{2}^{k-2} y_{3}^{k-3} \cdots y_{l-1}^{k-l+1} y_{l}^{k-l+1} y_{l+1}^{k-l} \cdots y_{k-2}^{3} y_{k-1}^{2} y_{k}^{N}$. We claim that this monomial survives in the right hand side of (2) for sufficiently 
large $N$ - say $N>\left(\begin{array}{c}k \\ 2\end{array}\right), N=2^{\nu}$. First note that since $N$ is large, the only monomials which involve $y_{k}^{N}$ must come from the terms $N_{i k} C_{i k}, 1 \leq i \leq l$. In $N_{i k}$ the only terms which contribute to the above monomial are $y_{k}^{N}\left(\sum_{\substack{p=1 \\ p \neq i}}^{k-1} y_{p}\right)$. It is not difficult to show that the above monomial occurs $(l-1)$-times in $y_{k}^{N}\left(\sum_{p=2}^{k-1} y_{p}\right) C_{1 k}$, and that it does not occur in $y_{k}^{N}\left(\sum_{\substack{p=1 \\ p \neq i}}^{k-1} y_{p}\right) C_{i k}$ for $i>1$. Since $l$ is even, it follows that the above monomial survives in $D \psi_{N+1}(\sigma)$. Hence $\psi_{N+1}(\sigma) \neq 0$.

Let $\mathcal{D}$ denote the ideal of decomposable elements in $Z_{*}(G)$. Let $\overline{\mathcal{K}}_{m}$ denote the $\mathbb{Z} / 2$-vector space $\mathcal{K}_{m} /\left(\mathcal{K}_{m} \cap \mathcal{D}\right)$. The dimension of $\overline{\mathcal{K}}_{m}$ is the number of $\mathbb{Z} / 2$-linearly independent indecomposable elements in $\mathcal{K}_{m}$.

Theorem 3.6. Let $2^{s-1}<n \leq 2^{s}, n \geq 3$. Let $d=\operatorname{dim}_{\mathbb{Z} / 2} \overline{\mathcal{K}}_{m}$. Then

(i) $d \geq n-2^{r}+2$, when $m=2^{r}-1, m \leq n$,

(ii) $d \geq n-2^{r}$, when $2^{r}<m+1<2^{r+1} \leq 2^{s}$, and $m$ is odd,

(iii) $d \geq n-m$, when $m$ is even, $2 \leq m<n$.

Proof. We shall only prove (ii), proofs for other parts being exactly analogous, and make use of Examples 3.3 and 3.4. Let $m$ be odd and $2^{r}<m+1<2^{r+1}, r \leq s-1$. Then $\left[H_{l, k}\right] \in \mathcal{N}_{*}$ is indecomposable where $k=2^{r}, l=m+1-k$. Note that $l \geq 2$, and that $k<n$. Now let $\sigma=\left[H_{l, k}, \phi_{S, T}\right]$ be as in Example 3.5. By Example 3.5, $\psi_{N+1}(\sigma) \in \mathbb{Z}_{2}\left[y_{1}, \ldots, y_{k}\right]$ is not zero. Suppose that $\psi_{N+1}(\sigma)=\sum_{r \geq 0} P_{r} y_{t}^{r}$, with $1 \leq t \leq k, P_{r} \in \mathbb{Z}_{2}\left[y_{1}, \ldots, y_{t-1}, y_{t+1}, \ldots, y_{k}\right]$, is the expression for $\bar{\psi}_{N+1}(\sigma)$ as a polynomial in $y_{t}$ with positive degree.

Now, for $1 \leq j \leq n-2^{r}$, let $A_{j}=\{\varnothing,\{1\}, \ldots,\{t-1\},\{t+1\}, \ldots,\{k\},\{k+j\}\}$, and let

$$
B_{j}= \begin{cases}T & \text { if } l<t, \\ T \cup\{k+j\} \backslash\{t\} & \text { if } t \leq l .\end{cases}
$$

Then, writing $\sigma_{j}=\left[H_{l, k}, \phi_{A_{j}, B_{j}}\right]$, we see that $\psi_{N+1}\left(\sigma_{j}\right)=\sum_{r \geq 0} P_{r} y_{k+j}^{r}$. Therefore, $\psi_{N+1}\left(\sigma+\sigma_{j}\right)=\sum P_{r}\left(y_{t}^{r}+y_{k+j}^{r}\right) \neq 0$. Hence by Proposition 3.1, it follows that $\sigma+\sigma_{j}$ is indecomposable. Clearly $\varepsilon_{*}\left(\sigma+\sigma_{j}\right)=2\left[H_{l, k}\right]=0$. Therefore $\sigma+\sigma_{j} \in \mathcal{K}_{m}$. Writing $u_{j}=\sigma+\sigma_{j}$, for $1 \leq j \leq n-2^{r}$, we see that for any sequence of $1 \leq j_{1}<\cdots<j_{p} \leq n-2^{r}$, one has $\psi_{N+1}\left(u_{j_{1}}+\cdots+u_{j_{p}}\right)=\sum_{1 \leq q \leq p} \psi_{N+1}\left(u_{j_{q}}\right)=$ $\sum P_{r}\left(p y_{t}^{r}+\sum_{1 \leq q \leq p} y_{k+j_{q}}^{r}\right) \neq 0$. This proves that $u_{1}, \ldots, u_{n-2^{r}}$ are linearly independent in $\bar{K}_{m}$, completing the proof.

Remark 3.7. Note that in the course of the above proof one could as well work with the smallest integer $M$ such that $\psi_{M}(\sigma) \neq 0$ in the place of the integer $N+1$.

The above theorem suggests the following conjecture. Note that the group $\operatorname{Aut}\left(\mathbb{Z}_{2}\right)^{n} \cong S L_{n}\left(\mathbb{Z}_{2}\right)$ acts on $Z_{*}(G)$ as $\mathbb{Z}_{2}$-algebra automorphisms. Indeed if $w \in S L_{n}\left(\mathbb{Z}_{2}\right)$, then $w([M, \phi])=\left[M, \phi^{w}\right]$, where $\phi^{w}(t, x)=\phi(w(t), x)$ for all $x \in M$. In particular, let $\sigma \in Z_{d}(G)$ be indecomposable. Then so is $w(\sigma)$, and $\sigma+w(\sigma) \in \mathcal{K}_{d}$.

Conjecture. If $w(\sigma) \neq \sigma$, then $\sigma+w(\sigma)$ is an indecomposable element in $\mathcal{K}_{d}$.

Let $u_{1}, u_{2}, \ldots, u_{k}$ be homogeneous elements in $Z_{*}(G)$. Assume that there exists an integer $N_{i}$ such that $f_{i}:=\psi_{N_{i}}\left(u_{i}\right) \neq 0$. We may suppose that $N_{i}$ is the smallest such integer. By relabelling the $u_{i}$ 's if necessary, we assume that $N_{1} \leq N_{2} \leq \cdots \leq$ 
$N_{k}$. Let $i_{0}=0<i_{1}<\cdots<i_{p}=k$ be such that $N_{j}=N_{i_{r}}$ for $i_{r-1}+1 \leq j \leq i_{r}$, $1 \leq r \leq p$.

Proposition 3.8. With the above notation, assume that $f_{1}, \ldots, f_{k} \in \mathbb{Z}_{2}\left[y_{1}, \ldots, y_{n}\right]$ are algebraically independent. Then $u_{1}, \ldots, u_{k}$ are algebraically independent.

Proof. Suppose that $P\left(u_{1}, \ldots, u_{k}\right)=0$ where $P$ is a homogeneous polynomial of degree $d$. Then we can write $P$ as $P=\sum \varepsilon_{\underline{r}} u_{1}^{r_{1}} \cdots u_{k}^{r_{k}}$ where the sum is over all sequences $\underline{r}=\left(r_{1}, \ldots, r_{k}\right) \in \mathbb{N}^{k}$ with $\sum_{i=1}^{k} r_{i}\left|u_{i}\right|=d, \varepsilon_{\underline{r}} \in\{0,1\}, \mathbb{N}$ being the set of non-negative integers. Let $|\underline{r}|=r_{1}+\cdots+r_{k}$. Let $S=\left\{\underline{r} \mid \varepsilon_{\underline{r}}=1\right\}$, and let $s=\min \{|\underline{\mid}| \mid \underline{r} \in S\}$. Note that $P=\sum_{r \in S} u_{1}^{r_{1}} \cdots u_{k}^{r_{k}}$. For $\underline{r} \in S$, let $\underline{r}^{j}=r_{i_{j-1}+1}+$ $\cdots+r_{i_{j}}, 1 \leq j \leq p$. Let $S_{p}=\{\underline{r} \in S|| \underline{r} \mid=s\}$, and let $s_{p}=\min \left\{\underline{r}^{p} \mid \underline{r} \in S_{p}\right\}$. Similarly, let $S_{p-1}=\left\{\underline{r} \in S_{p} \mid \underline{r}^{p}=s_{p}\right\}$, and set $s_{p-1}=\min \left\{\underline{r}^{p-1} \mid \underline{r} \in S_{p-1}\right\}$. Having defined $S_{p}, S_{p-1}, \ldots, S_{p-j+1}$ and $s_{p}, s_{p-1}, \ldots, s_{p-j+1}$, we define $S_{p-j}$ and $s_{p-j}$ as $S_{p-j}=\left\{\underline{r} \in S_{p-j+1} \mid \underline{r}^{p-j+1}=s_{p-j+1}\right\}$ and $s_{p-j}=\min \left\{\underline{r}^{p-j} \mid \underline{r} \in S_{p-j}\right\}$. Note that $S_{1}$ consists precisely of those $\underline{r} \in S_{p}$ such that the sequence $\left(\underline{r}^{p}, \ldots, \underline{r}^{1}\right)=$ $\left(s_{p}, \ldots, s_{1}\right)$ is the smallest in the lexicographic ordering of $\mathbb{N}^{p}$, as $\underline{r}$ varies in $S_{p}$. Now let

$$
\lambda=N_{i_{1}}^{s_{1}} \ldots N_{i_{p}}^{s_{p}}=\underbrace{N_{i_{1}}, \ldots, N_{i_{1}}}_{s_{1}}, \cdots, \underbrace{N_{i_{p}}, \ldots, N_{i_{p}}}_{s_{p}} .
$$

Claim. For $\underline{r} \in S$,

$$
\psi_{\lambda}\left(u_{1}^{r_{1}} \cdots u_{k}^{r_{k}}\right)= \begin{cases}f_{1}^{r_{1}} \cdots f_{k}^{r_{k}} & \text { if } \underline{r} \in S_{1}, \\ 0 & \text { otherwise. }\end{cases}
$$

It is easily seen that $\psi_{\lambda}\left(u_{1}^{r_{1}} \cdots u_{k}^{r_{k}}\right)=0$ if $|\underline{r}|>s=|\lambda|$. Also it is trivial to see that $\psi_{N_{i_{j}}^{s_{j}}}\left(u_{i_{j-1}+1}^{r_{j-1}+1} \cdots u_{i_{j}}^{r_{j}}\right)=f_{i_{j-1}+1}^{r_{j-1}+1} \cdots f_{i_{j}}^{r_{j}}, 1 \leq j \leq p$. Furthermore $\psi_{M_{1} \cdots M_{q}}\left(u_{i_{j-1}+1}^{r_{1}} \cdots u_{i_{j}}^{r_{q}}\right)=0, q=i_{j}-i_{j-1}$, if some $M_{t}<N_{i_{j}}$ by minimality of $N_{j}$. By Lemma 3.2 the claim follows. To complete the proof of the lemma, we apply $\psi_{\lambda}$ to $P\left(u_{1}, \ldots, u_{k}\right)=0$. By the claim above

$$
0=\psi_{\lambda}\left(P\left(u_{1}, \ldots, u_{k}\right)\right)=\sum_{\underline{r} \in S_{1}} \psi_{\lambda}\left(u_{1}^{r_{1}} \cdots u_{k}^{r_{k}}\right)=\sum_{\underline{r} \in S_{1}} f_{1}^{r_{1}} \cdots f_{k}^{r_{k}}
$$

which contradicts the hypothesis that the $f_{i}$ 's are algebraically independent.

Let $u_{1}, \ldots, u_{r}$ be homogeneous elements in $\mathcal{K}_{*}$ constructed as in the proof of Theorem 3.6. By Remark 3.7 there exist $M_{i}$ smallest such that $\psi_{M_{i}}\left(u_{i}\right) \neq 0$. Write $\psi_{M_{i}}\left(u_{i}\right)=P_{i}\left(y_{1}, \ldots, y_{k_{i}}\right)$ where $P_{i}$ involves $y_{k_{i}}$.

Theorem 3.9. With the above notation, suppose $u_{1}, \ldots, u_{r} \in \mathcal{K}_{*}$ are such that $1 \leq k_{1}, \ldots, k_{r} \leq n$ are all distinct. Then $u_{1}, \ldots, u_{r}$ are algebraically independent.

Proof. The theorem follows immediately from the above proposition as $\psi_{M_{i}}\left(u_{i}\right)=$ $P_{i}\left(y_{1}, \ldots, y_{k_{i}}\right), i=1, \ldots, r$, are clearly algebraically independent.

In particular, it follows from Theorem 3.6 that $\mathcal{K}_{m}$ has at least $r$ elements which generate a polynomial algebra on $r$ variables where

(i) $r=n-2^{q}+2$, when $m=2^{q}-1, m \leq n$,

(ii) $r=n-2^{q}$, when $2^{q}<m+1<2^{q+1} \leq 2^{s}$, and $m$ is odd,

(iii) $r=n-m$, when $m$ is even, $2 \leq m<n$. 
We conclude this section with the following questions. Let as usual $G=\left(\mathbb{Z}_{2}\right)^{n}$.

Question 1. Is $Z_{*}(G)$ finitely generated as a $\mathbb{Z}_{2}$-algebra?

Question 2. Are there indecomposable elements in $Z_{*}(G)$ beyond dimension $2^{n}-$ 2 ?

\section{TANGential RePresentations}

An important step in determining the structure of $Z_{*}\left(\mathbb{Z}_{2}^{2}\right)$ is the following observation of Conner and Floyd (Lemma 32.3, [1]). Let $(M, \phi)$ be a smooth closed $\mathbb{Z}_{2} \times \mathbb{Z}_{2}$-manifold, and let $\alpha \subset\{1,2\}$. If $Y_{\alpha}$ occurs in the representation $T_{x} M$ with multiplicity $p$, at some stationary point $x$. Then it occurs with the same multiplicity at an even number of stationary points. We shall show in this section that this phenomenon happens for any $\left(\mathbb{Z}_{2}\right)^{n}$-manifold with finite stationary point set.

Theorem 4.1. Let $n \geq 2$ and $(M, \phi)$ be a closed $\left(\mathbb{Z}_{2}\right)^{n}$-manifold with finite stationary point set. Suppose that for some stationary point $x$, and $\alpha \subset \underline{n}, Y_{\alpha}$ occurs with multiplicity $p$ in $T_{x} M$. Then it occurs with the same multiplicity at an even number of stationary points.

Proof. Let $d=\operatorname{dim} M$. We use the notation of section 3 . Let $V=Y_{\alpha_{1}}^{p_{1}} \cdots Y_{\alpha_{k}}^{p_{k}}$, $\sum p_{i}=d, \alpha_{1}, \ldots, \alpha_{k}$ distinct. Then

$$
\gamma(V)=\frac{1}{y_{\alpha_{1}}^{p_{1}} \cdots y_{\alpha_{k}}^{p_{k}}}\left(1+b_{1} y_{\alpha_{1}}+b_{2} y_{\alpha_{1}}^{2}+\cdots\right)^{p_{1}} \cdots\left(1+b_{1} y_{\alpha_{k}}+b_{2} y_{\alpha_{k}}^{2}+\cdots\right)^{p_{k}} .
$$

Let $r<d$. The coefficient $C_{d-r}(V)$ of $b_{1}^{d-r}$ in the expression for $\gamma(V)$ can be calculated as follows: If $p_{1}=: p \geq r$, then

$$
C_{d-r}(V)=\frac{\left(\begin{array}{l}
p \\
r
\end{array}\right)}{y_{\alpha_{1}}^{r}}+P_{\alpha_{1}}(V)
$$

where $P_{\alpha_{1}}(V)$ is a polynomial in $\left(1 / y_{\alpha_{1}}\right)$ of degree less than $r$ with coefficients in $\mathbb{Z}_{2}\left[1 / y_{\alpha_{j}} \mid 2 \leq j \leq k\right]$. If $p<r$, then $C_{d-r}(V)=Q_{\alpha_{1}}(V)$, a polynomial in $\left(1 / y_{\alpha_{1}}\right)$ of degree less than $r$ with coefficients in $\mathbb{Z}_{2}\left[1 / y_{\alpha_{j}} \mid j \neq 1\right]$. Let $S_{\alpha, r}=\left\{x \in S \mid Y_{\alpha}\right.$ occurs with multiplicity $r\}$. Fix $\alpha=\alpha_{1}$. We need to show that $\left|S_{\alpha, r}\right|$ is even for each $r, 1 \leq r \leq d$. Now suppose that $r=s$ is the largest integer such that $Y_{\alpha}$ occurs with multiplicity $s$, among $T_{x} M$, as $x$ varies over the (finite) set $S$ of stationary points. One knows that $s<d$. Then

$$
0=\underbrace{\psi_{1, \ldots, 1}}_{d-s}(M, \phi)=\sum_{x \in S} C_{d-s}\left(T_{x} M\right)
$$

Multiplying both sides of (3) by $y_{\alpha}^{s}$ we obtain

$$
0=\sum_{x \in S_{\alpha, s}}\left(1+y_{\alpha}^{s} P_{\alpha}\left(T_{x} M\right)\right)+\sum_{x \in S \backslash S_{\alpha, s}} y_{\alpha}^{s} Q_{\alpha}\left(T_{x} M\right) .
$$

Note that $y_{\alpha}^{s} P_{\alpha}\left(T_{x} M\right), y_{\alpha}^{s} Q_{\alpha}\left(T_{x} M\right)$ are polynomials in $y_{\alpha}$ without constant terms having coefficients in $\mathbb{Z}_{2}\left[1 / y_{\beta} \mid \beta \neq \alpha\right]$. Clearing the denominators in (4), we obtain 
an expression

$$
\begin{array}{r}
0=\left(\sum_{x \in S_{\alpha, s}} 1\right) y_{\beta_{1}}^{k_{1}} \cdots y_{\beta_{t}}^{k_{t}}+\left(\sum_{x \in S_{\alpha, s}} y_{\alpha}^{s} P_{\alpha}\left(T_{x} M\right)\right) y_{\beta_{1}}^{k_{1}} \cdots y_{\beta_{t}}^{k_{t}} \\
+\left(\sum_{x \in S \backslash S_{\alpha, s}} y_{\alpha}^{s} Q_{\alpha}\left(T_{x} M\right)\right) y_{\beta_{1}}^{k_{1}} \cdots y_{\beta_{t}}^{k_{t}}
\end{array}
$$

in $\mathbb{Z}_{2}\left[y_{1}, \ldots, y_{n}\right]$, where $\beta_{1}, \ldots, \beta_{t} \in \mathcal{P}(\underline{n}), \alpha \neq \beta_{i}, 1 \leq i \leq t, k_{1}, \ldots, k_{t}>0$. Put $y_{\alpha}=0$ in the above expression. Then we obtain $0=\left|S_{\alpha, s}\right| \cdot y_{\beta_{1}}^{k_{1}} \cdots y_{\beta_{t}}^{k_{t}}$ in $\mathbb{Z}_{2}\left[y_{1}, \ldots, y_{n}\right] /\left\langle y_{\alpha}\right\rangle$. As $\mathbb{Z}_{2}\left[y_{1}, \ldots, y_{n}\right] /\left\langle y_{\alpha}\right\rangle$ is again a polynomial algebra in $(n-1)$ variables, we see that $\left|S_{\alpha, s}\right|$ must be even.

Inductively assume that $Y_{\alpha}^{p}$ occurs in $T_{x} M$, if at all, at an even number of stationary points for any $d>p>r$. We must show that $Y_{\alpha}^{r}$ occurs in $T_{x} M$, if at all, for an even number of stationary points. Proceeding exactly as before we see that

$$
0=\sum_{p \geq r}\left(\sum_{x \in S_{\alpha, p}}\left(\begin{array}{l}
p \\
r
\end{array}\right)+y_{\alpha}^{r} P_{\alpha}\left(T_{x} M\right)\right)+\sum_{x \in S \backslash \bigcup_{p \geq r} S_{\alpha, p}} y_{\alpha}^{r} Q_{\alpha}\left(T_{x} M\right) .
$$

Again note that $y_{\alpha}^{r} P_{\alpha}\left(T_{x} M\right)$ and $y_{\alpha}^{r} Q_{\alpha}\left(T_{x} M\right)$ are polynomials in $y_{\alpha}$ without constant terms and having coefficients in $\mathbb{Z}_{2}\left[1 / y_{\beta} \mid \beta \neq \alpha\right]$. As before we see that $\sum_{p \geq r}\left|S_{\alpha, p}\right|\left(\begin{array}{c}p \\ r\end{array}\right)=0(\bmod 2)$. Hence $\left|S_{\alpha, r}\right| \equiv \sum_{p>r}\left|S_{\alpha, p}\right|\left(\begin{array}{l}p \\ r\end{array}\right) \equiv 0(\bmod 2)$, since by the induction hypothesis each $\left|S_{\alpha, p}\right|, p>r$, is even.

Remark 4.2. It may be noted that our method can be used to interpret the vanishing of $\psi_{\lambda}(M, \phi)$ for $|\lambda|<d=\operatorname{dim} M$ in terms of the irreducible representations $Y_{\alpha}, \alpha \subset \underline{n}$, which occur in $T_{x} M$ as $x$ varies in the (finite) set of stationary points of $(M, \phi)$.

\section{REFERENCES}

1. P. E. Conner, Differentiable periodic maps, 2nd Ed., L.N.M. (738) Springer-Verlag, 1979. MR 81f:57018

2. P. E. Conner and E. E. Floyd, Differentiable periodic maps, Ergebnisse Sr-33, Springer-Verlag, 1964. MR 31:750

3. T. tom Dieck, Fixpunkte vertauschbarer involutionen, Archiv der math., 20, 295-298, 1969. MR 42:3798

4. 1 , Characteristic numbers of $G$ manifolds. I, Invent. Math. 13, 213-224, 1971. MR 46:8236

5. C. Kosniowski and R. E. Stong, $\left(\mathbb{Z}_{2}\right)^{k}$-Actions and Characteristic numbers, Indiana Univ. Math. J., 28, 725-743, 1979. MR 81d:57027

6. K. Y. Lam, A formula for the tangent bundle of flag manifolds and related manifolds, Trans. Amer. Math. Soc., 213, 305-314, 1975. MR 55:4196

7. J. W. Milnor and J. D. Stasheff, Characteristic classes, Ann. Math. Stud., 76, Princeton, 1974. MR 55:13428

8. G. Mukherjee, Equivariant cobordism of Grassmann and flag manifolds, Proc. Ind. Acad. Sci., 105, 381-391, 1995. MR 97g:57045

9. P. Sankaran, Determination of Grassmann manifolds which are boundaries, Canad. Math. Bull., 34, 119-122, 1991. MR 92h:57049

10. P. Sankaran and K. Varadarajan, Group actions on flag manifolds and cobordism, Canad. J. Math., 45, 650-661, 1993. MR 94k:57050 
11. R. E. Stong, Equivariant bordism and $\left(\mathbb{Z}_{2}\right)^{k}$-actions, Duke Math. J. 37, 779-785, 1970. MR 42:6847

12. R. E. Stong (reviewer). MR 89d:57050

Stat-Math Division, Indian Statistical Institute, 203 B. T. Road, Calcutta-700 035, INDIA

E-mail address: goutam@isical.ernet.in

SPIC Mathematical Institute, 92 G. N. Chetty Road, Madras-600 017, India

E-mail address: sankaran@smi.ernet.in 